\title{
Challenges and Management in Gynaecological Cancers in Covid 19 Crises: An Institutional Experience
}

\author{
Sumit Kumar ${ }^{3}$, Vishal Kaundal ${ }^{2}$, Shilpa Sood ${ }^{6}$, Vandana Singh \\ Kushwaha $^{5}$, Shalini Verma ${ }^{1 *}$, Muninder Negi ${ }^{1}$ and Nitin Gupta ${ }^{4}$ \\ ${ }^{1}$ Radiation Oncology Department, Dr RPGMC Tanda, India \\ ${ }^{2}$ Surgery Department, Dr RPGMC Tanda, India \\ ${ }^{3}$ Radiation Department, AIIMS, NEW Delhi, India \\ ${ }^{4}$ Nuclear Medicine Department, Dr RPGMC Tanda, India \\ ${ }^{5}$ Radiation Oncology Vydehi Institute of Medical Sciences and Research, India \\ ${ }^{6}$ Gynaecology, Lady Harding, India \\ *Corresponding Author: Shalini Verma, Radiation Oncology Department, \\ Dr RPGMC Tanda, India.
}

Received: October 23, 2020

Published: November 18, 2020

(C) All rights are reserved by Shalini Verma., et al.

\begin{abstract}
Objective: To illustrate our institutional experience about the challenges we faced and steps taken in the management of the patients with gynaecological malignancies during Covid-19 crises at Dr. R.P.G.MC Tanda.

Methods: The recommendations and guidelines framed by the World Health Organization and other international authorities were followed.

Results: With our multidisciplinary approach and intensive efforts we tried to provide all the feasible services to the ones requiring urgent and active treatment while at the same time protecting the ones (from Covid-19 infection ) not in immediate need of treatment by postponing their visits and treatment.

Conclusion: Covid 19 pandemic has resulted in unprecedented global healthcare crises. Our institution has illustrated the challenges faced in managing patients with gynaecological cancers. Due to the fear of increased risk of Covid-19 infection in the cancer patients which would result in devastating complications and very poor outcome, the preventive steps taken resulted in appreciable upstaging and progression of disease on imaging and blood investigations.
\end{abstract}

Keywords: COVID-19; Pandemic; Gynaecological Cancer Care

\section{Introduction}

Coronavirus disease is highly contagious disease caused by a strange type of virus to which humans have never been exposed before. This rapidly growing pandemic has posed tremendous strain on healthcare services [1]. In this overwhelming situation the health care system had to make immediate adaptation, delaying of the treatment being the most common, thereby, raising the concern about cancer, especially gynaecological caners. As gynaecological cancers being the most common cancer in women are on continuous rise during the pandemic. The majority of gynaecological cancers are already diagnosed at advanced stages and further delay would compromise outcomes. Hence, management of gynaecological cancers is quite challenging for both the oncologists and the patients due to restricted hospital visits to prevent the spread of COVID-19 infection.

Thus, keeping in mind the current scenario the multidisciplinary approach to minimize the morbidity, mortality and resultant com- 
plications from anticancer treatment made, and at the same time measures to protect the patient and staff should be taken.

At present various management guidelines have been framed regarding continuation or delaying of cancer treatment and decision should be taken after balancing the risk associated with exposure to Covid-19 infection and cancer treatment.

Our study was conducted to illustrate the challenges we faced while managing gynaecological cancers in period of Covid-19 Crises by diverting our treatment from the standard ones, but we found poor outcome in the patients when they returned back.

\section{Methodology}

Why there is need to worry much about cancer patients in Covid-19 Crises

Cancer patients are usually immunocompromised due to malignancy, previous recent or ongoing treatment and have higher risk of contracting Covid 19 infection resulting in rapid deterioration.

Cancer acts as independent prognostic factor irrespective of age, comorbidities or addiction.

There was fatal outcome (75\% vs $43 \%$ ) in the patients with history of surgery or chemotherapy in the past one month [2].

One report from China revealed that two women with gynaecological cancer were cured of pneumonia while one died of Covid infection. This increased susceptibility to Covid 19 infection along with rapid deterioration in gynaecological cancer has left us with anxiety [3].

Moreover, increased need of ICU admission and ventilator dependency in cancer patients have further worsened the situation.

In the present situation, there is delay in cancer diagnosis, treatment accessibility and actual treatment administration leading to devastating consequences.

\section{Impact of delayed surgery in cervical cancer}

In the study conducted in Taiwan, 1 and 5 year survival in cervical cancer (all stages) patients was poor for those who received treatment after 4 months of diagnosis in comparison to those who received timely treatment (1-year survival: $91 \%$ vs. $60 \%$, 5-year survival: $71 \%$ vs. $38 \%$ ) [4].
The impact of radiation delay in cervical cancer

Treatment prolongation has negative impact on tumour outcome due to repopulation of tumour cells [5]. The study by Fyles., et al. on 800 patients in stage 1-IV cervical cancer (median treatment time -36 days) found that there was $1 \%$ loss of pelvic control with each one day treatment delay beyond median [6]. Similarly, in another retrospective study by Petereit., et al. [7] on 209 patients with stage IB-IIIB cervical cancer, the median duration of treatment was 55 days. They concluded pelvic control of $87 \%$ versus $54 \%$ in patients with treatment completion in 55 days and beyond it respectively.

The recent study by Tanderup., et al. [8] on 488 patients with locally advanced cervical cancer treated with chemotherapy and EBRT plus image-guided brachytherapy showed that overall treatment time of more than 7 weeks resulted in 3-year overall local control (LC) of 86\% - 94\%. It was found that additional 5 Gy was required to compensate for loss of LC when treatment was extended beyond 7 weeks.

Ideally whole treatment (chemotherapy, external beam radiotherapy and brachytherapy) should be completed within 8 weeks. External beam radiotherapy should begin 4 - 6 weeks after surgery with minimum treatment interruptions, but not after 12 weeks [9].

Adjuvant chemoradiation should be started after 4 - 6 weeks after surgery but not after 8 weeks [10].

\section{Impact of delayed surgery in endometrial cancer}

In another study by Shalowitz., et al. [11] timing of surgery when done in third week after diagnosis has great impact on 5-year survival in both low and high risk endometrial cancer patients. (Lowrisk disease: 87.4\%; high risk disease 66.9\%). The patients with low-risk endometrial disease had increased risk of death with surgery is delayed to after 8 weeks of diagnosis and is $16 \%$ inferior if performed after 4 months of diagnosis than in patients with surgery within 3 weeks. In contrast to this high-risk endometrial cancer patients delayed surgery to more than 21 weeks had no impact on survival [11].

\section{Impact of radiation delay in Ca endometrium}

The study by Ahmed., et al. [12] found decreased disease-specific survival in patients with delay in initiation of radiotherapy by more than 6 weeks after surgery. Similarly, another study by Fabrini., et al. [13] stated increased rate of local recurrence with 
interval of more than 9 weeks between surgery and starting of radiotherapy. Ideally vaginal brachytherapy should be started within 8 weeks of surgery, but not more than 12 weeks.

\section{Impact of delayed treatment in ovarian cancer}

As majority diagnosed in advanced stages, further treatment delay results in unfavourable outcome. In the meta-analysis by Liu., et al. [14] worse overall survival was found in patients whose adjuvant chemotherapy administration was delayed by more than 6 weeks.

\section{Concern regarding chemotherapy and radiotherapy}

Due to lack of specific guidelines concept regarding initiating or delaying chemotherapy for another 6-8 weeks remains unclear. The patients should be explained about the increased susceptibility of acquiring Covid-19 infection, higher risks of associated adverse effects like neutropenia and lymphopenia with chemotherapy and the risk of rapid deterioration if they acquire Covid-19 infection.

Thus, decision should be made after taking all these things into consideration. The newly diagnosed cases can be started on neoadjuvant chemotherapy (NACT). For the patients already on NACT in whom surgery is not feasible after 3 - 4 cycles, it may be continued till complete six cycles $[15,16]$.
However, chemotherapy need to be postponed in view of increasing number of cases. The initiation of adjuvant radiotherapy within 6 weeks of surgery also acts as prognostic factor, but due to lack of proper recommendations during this period of crises, physician has to individualize its use and postpone the treatment as needed. Hypofractionation should be tried wherever possible $[15,16]$.

\section{Fear of initiating the treatment on time}

On one side of detrimental outcomes with delayed treatment. There is also 3.5 times more fear of need of increased hospitalization, ventilator support and death if they acquire Covid-19 infection which is hampering to initiate and administer adequate treatment on time. Keeping all these things in mind patients should be prioritized into those requiring urgent treatment or in whom treatment could be delayed.

It is also very challenging to counsel the patients regarding the need to delay treatment and the associated outcomes with delayed treatment and their increased risk of Covid-19 infection.

Another challenge is the limited supply and resources along with limited Covid-19 testing and ICU provision in medical institutions and ensuring the safety of health care workers $[17,18]$.

Steps taken in our institute to decrease rush in the outpatient care

\begin{tabular}{|c|c|}
\hline Purpose & Measures \\
\hline Reducing the risk of patients exposure & $\begin{array}{l}\text { The visits were restricted to the patients on } \\
\text { Active treatment/requiring urgent attention } \\
\text { All the routine follow-ups were postponed } \\
\text { And teleconsultation was started } \\
\text { All unnecessary interventions like routine imaging, blood tests, } \\
\text { serum markers in asymptomatic patients were postponed } \\
\text { Limiting the number of patients in waiting area } \\
\text { Posters pasted in waiting area regarding social distancing }\end{array}$ \\
\hline Reducing the exposure to the staff & $\begin{array}{c}\text { Only patients were allowed inside the OPDs } \\
\text { Only one attendant (uninfected) was allowed to accompany the } \\
\text { patient outside OPDs } \\
\text { To minimize the exposure to the staff, roster was made in which } \\
\text { healthcare workers and physicians were not called daily and were } \\
\text { assigned specific duty days } \\
\text { Reducing the number of medical staff } \\
\text { Reducing the number of nursing staff } \\
\text { Reducing the number of staff at reception } \\
\text { All the staff to wear gowns, masks and gloves }\end{array}$ \\
\hline
\end{tabular}

\section{Table}

Citation: Shalini Verma., et al. "Challenges and Management in Gynaecological Cancers in Covid 19 Crises: An Institutional Experience". Acta Scientific Cancer Biology 4.12 (2020): 03-09. 
Strategies to manage gynaecological cancers in Covid -19 era: Cervical cancer

As per recommendations by American Society for colposcopy and cervical Pathology [15].

Preinvasive disease

- $\quad$ LSIL (Low-grade squamous intraepithelial lesion) - Diagnostic evaluation can be postponed up to 6-12 months.

- $\quad$ HSIL (High-grade Squamous intraepithelial lesion) - Diagnostic evaluation can be postponed up to 3 months.

Early stage disease

If the available resources allow surgery without compromising general principles, proceed for surgery. The procedures can be postponed for 6 - 8 weeks or till the completion of healthcare crises.

Simple trachelectomy with or without sentinel lymph node dissection or conization can be done in low risk patients with tumour of less than $2 \mathrm{~cm}$.

NACT can be considered in patients with gross disease. Definitive Concurrent Chemoradiation should be done in early stage cervical cancers where radical hysterectomy is the standard of care.

Locally advanced disease

Concurrent Chemoradiotherapy should be administered whenever possible. Hypofractionation can be considered when feasible. Brachytherapy should be given in the same way as before without any gaps.

For patients below 70 years without any comorbidities

- Concurrent weekly cisplatin is the standard of care.

- $\quad$ Chemotherapy may be omitted as per the available resources.

For patients above $\mathbf{7 0}$ years with/without comorbidities

- Pelvic radiotherapy only without chemotherapy

- Using small pelvic radiotherapy portal

\section{Metastatic disease}

The asymptomatic or low-burden disease patients, or older patients with poor performance status and associated comorbidities. It is advisable to delay intravenous chemotherapy. Oral metro- nomic therapy can be considered when feasible. For symptomatic patients with high-burden disease and good performance status, palliative radiotherapy or single -agent carboplatin is preferred till the crises is over, metronomic therapy is another option.

Second line or beyond

Further chemotherapy is not beneficial. Hence, oral metronomic therapy or to delay therapy is an option.

Endometrial cancer [15]

Low-risk disease

- Intrauterine device or hormonal therapy should be considered for Grade- 1 lesions.

\section{High-risk disease}

- Total abdominal hysterectomy with bilateral salpingo-oophorectomy is the standard treatment.

- Adjuvant radiotherapy is given less priority and can be delayed when not feasible.

The decision regarding adjuvant treatment should be left on patient's willingness after explaining the risk-benefit ratio in this pandemic situation. Multiagent chemotherapy can be avoided.

\section{Metastatic disease}

The asymptomatic and patients with low-burden disease, or older with comorbidities and poor PS, intravenous chemotherapy can be delayed and endocrine therapy can be given. The symptomatic patients with high-burden disease and good PS, single-agent carboplatin is with endocrine therapy as an alternative is preferred till the crises subsides

\section{Second line or beyond}

Not much benefit with chemotherapy. The endocrine therapy or treatment delay are the options.

Ovarian cancer [15]

Early stage disease

The standard upfront surgery requires long standing surgery with multi-visceral resection and may need postoperative intensive care unit, a challenging situation in current scenario. Thus, NACT should be used in both early and advanced disease. NACT should be extended to complete six cycles rather than three. Secondary debulking is avoided in recurrent settings. 
The use of filgrastim should be encouraged to decrease neutropenia. Bevacizumab should be used carefully owing to great toxicity.

\section{Adjuvant chemotherapy}

Intraperitoneal chemotherapy should be avoided. Adjuvant chemotherapy should be started within 4-6 weeks of surgery and should be given in all cases. Paclitaxel and carboplatin is the preferred regimen in view of lack of data on single agent platinum $[19,20]$. Dose-dense weekly paclitaxel and carboplatin once three weekly should be avoided. Bevacizumab and maintenance therapy should be avoided.

\section{Advanced disease}

Palliative chemotherapy should be considered in all patients when feasible. Depending upon patient's age, PS, comorbidities single-agent carboplatin or combination of paclitaxel and carboplatin should be used.

\section{Platinum-sensitive relapse}

Intravenous chemotherapy can be delayed in old, asymptomatic, poor PS, low-burden diseased and comorbid patients. Oral metronomic therapy is another option.

The patients with symptomatic, high-burden disease, good PS, single agent carboplatin or paclitaxel and carboplatin in combination are given. Poly ADP ribose polymerase inhibitors after 4 cycles can be considered.

\section{Platinum-resistant relapse}

Chemotherapy is generally avoided in asymptomatic cases. The symptomatic patients are managed symptomatically or metronomic therapy can be started. Chemotherapy can be delayed in patients with low-grade cancers or non-endometroid and non-serous histology's and endocrine therapy should be prescribed in patients with bulky disease.

\section{Vulva}

Early cases should not be postponed. Advanced cases requiring extensive resections should be treated with chemotherapy and radiotherapy. In metastatic settings, chemotherapy should be considered $[15,21]$.

\section{Vaginal cancers}

The patients mostly present in advanced stages for which chemotherapy and radiotherapy should be considered $[15,21]$.

\section{Trophoblastic tumours}

These tumours should be treated with systemic chemotherapy [21].

Our institute followed the steps wherever feasible in manging gynaecological cancer patients.

\section{Precautions taken in the radiation Zone by our institute}

To protect both the patients and staff, weekly team rotation was done, thereby, reducing the number of workers at this period of crisis. The patients already on treatment, finishing the treatment was the priority while delaying radiation for others. To avoid overcrowding only patients thermal screening and after asking about the symptoms related to Covid-19, were allowed to enter while keeping attendant outside the main door.

Patients were treated in dedicated time slots with staff wearing the protective equipment. The treatment couch was always sanitized after treatment of each patient. The posters regarding measures taken for self care were posted on the walls in hindi and were advised to read them. The whole area was properly decontaminated and sanitized daily.

Precautions taken in day care zone by our institute

All the patients were allowed to enter the department after taking pass from Flu Clinic where the symptoms regarding the infection were asked. Only single entry and exit was kept with one person entering/leaving at a time.

To limit the number of hospital visits some regimens were switched to the possible oral preparations. The patients with stable disease for more than 6 months, further treatment was stopped or delayed. A temporary break was given for slow growing metastatic disease. Special attention was given to Covid-19 symptoms to the patients on active treatment.

\section{Results and Discussion}

Our institute tried its best to deal with this pandemic with proper counselling the patients, limiting the number and visits of patients, dealing with the urgent cases and delaying the treatment in those not requiring urgent attention.

We followed proper the recommendation and guidelines in treating our gynaecological cancer patients.

In our study we have illustrated the challenges we had to face in managing gynaecological cancers, we found that almost $80 \%$ of 
patients of gynaecological cancers had disease progression which was appreciable on imaging scans (CT/MRI/PET) and on serum markers, another $10 \%$ were lost to follow up. Hence, we can say that due to complete lockdown and limited resources, we had no other alternative than to delay or modify our treatment options as per guidelines to protect our patients from Covid-19 as well. This delayed treatment had significant negative impact on patient outcome.

\section{Conclusion}

This coronavirus pandemic is quite challenging to the healthcare system. The has resulted in global healthcare crises with major effect on ICU, emergency department, and other healthcare specialities especially oncology department. Moreover, steps taken to prevent the transmission of virus further hampers treatment administration which has very bad impact on cancer patients.

Oncology patients should be given attention as these are more susceptible to Covid-19 infection. Alternative measures while safeguarding the patients should be taken. Further, delay in treatment results in poor outcome and disease progression. Therefore, with rapid increase in the number of cases gynaecological oncologists should follow balanced, compassionate and safe care to the patients.

\section{Bibliography}

1. Docea AO., et al. "A new threat from from an old enemy: Reemergence of coronavirus (Review)". International Journal of Molecular Medicine 45 (2020): 1631-1643.

2. Liang W., et al. "Cancer patients in SRAS-CoV-2 infection: A Nationwide analysis in China". Lancet Oncology 21.3 (2020): 335-337.

3. Zhang J., et al. "Management strategies for three patients with gynaecological malignancies during the outbreak of COVID19". Zhonghua fu Chan ke za zhi 55.4 (2020): 221-226.

4. Shen SC., et al. "Factors involved in the delay of treatment initiation for cervical cancer patients: A nationwide populationbased study". Medicine 95.33 (2016): e4568.

5. Allam JA., et al. "The effect of the overall treatment time of fractionated-irradiation on the tumour-control probability of a human sofe-tissue sarcoma xenograft in nude-nice". International Journal of Radiation Oncology 32 (1995): 105-111.

6. Fyles A., et al. "The effect of treatment duration in the local control of cervix cancer". Radiotherapy Oncology 25 (1992): 273-279.
7. Petereit DG., et al. "The adverse effect of treatment prolongation in cervical cancer". International Journal of Radiation Oncology • Biology • Physics 32 (1995): 1301-1307.

8. Tandrup K., et al. "Effect of tumor dose, volume and overall treatment time on local control after radiochemotherapy including MRI guided brachtherapy of locally advanced cervical cancer". Radiotherapy Oncology 120 (2016): 441-446.

9. Sedlis A., et al. "A randomized trial of pelvic radiation therapy versus no further therapy in selected pateints with stage IB carcinoma of the cervix after radical hystrectomy and pelvic lymphadenectome: A gynaecologic Oncology Group Study". Gynecology and Oncology 73 (1999): 177-183.

10. Peters WA., et al. "Concurrent Chemotherapy and pelvic radiation in high risk early stage cancer of the cervix". Journal of Clinical Oncology 18 (2000): 1606-13.

11. Shalowitz DI., et al. "Survival implications of time of endometrial cancers". American Journal of Obstetrics and Gynecology 216.3 (2017): 268e1-268e18.

12. Ahmad NR., et al. "Postoperative radiation therapy for surgically staged endometrial cancer- Impact of time (overall tretament time and surgery to radiation interval on outcome". International Journal of Radiation Oncology 33 (1995): 837-842.

13. Fabrini MG., et al. "Relationship between interval from surger to radiotherapy and local recurrence rate in patients with endometroid type endometrila cancer: A retrospective monoinstitutional Italian Study". Anticancer Research 32 (2012): 169-173.

14. Liu Y., et al. "Relationship between initiation time of adjuvant chemotherapy and survival in ovarian cancer patients: A doseresponse meta-analysis of cohort studies". Scientific Report 7.1 (2017): 1-8.

15. Ramirez PT., et al. "COVID-19 pandemic: options for management of gynecologic cancers". International Journal of Gynecological Cancer 30.5 (2020): 561-563.

16. Udeta M., et al. "Managing cancer care during the COVID-19 pandemic: agility and collaboration toward a common goal". Journal of the National Comprehensive Cancer Network 20 (2020): 1-4.

17. Huang C., et al. "Clinical features of patiens infected with 2019 novel coronavirus in Wuhan, China". Lancet 395.10223 (2020): 497-506.

18. Zhou FD., et al. "Clinical course and risk factors for mortality of adults in patients with COVD-19 in Wuha, china: A retrospective study". Lancet 395.10229 (2020): 1054-1062. 
19. Sapkota S., et al. "Ovarian cancer practice survey from the South Asian Association for Regional Cooperation (SAARC) Nations". Cancer Research, Statistics, and Treatment 2 (2019): 158-162.

20. Kaur S and Singh R. "Patterns of care for ovarian cancer". Cancer Research, Statistics, and Treatment 2 (2019): 217-220.

21. Akladios C., et al. "Recommendations for surgical management of gynecological cancers during the COVID-19 pandemic". Journal of Gynecology Obstetrics and Human Reproduction 49.6 (2020): 101729.

\section{Assets from publication with us}

- Prompt Acknowledgement after receiving the article

- Thorough Double blinded peer review

- Rapid Publication

- Issue of Publication Certificate

- High visibility of your Published work

Website: www.actascientific.com/

Submit Article: www.actascientific.com/submission.php

Email us: editor@actascientific.com

Contact us: +919182824667 